\title{
Solving the Dirichlet problem constructively
}

\author{
D.S. BRIDGES \\ M. MCKUBRE-JORDENS
}

\begin{abstract}
The Dirichlet problem is of central importance in both applied and abstract potential theory. We prove the (perhaps surprising) result that the existence of solutions in the general case is an essentially nonconstructive proposition: there is no algorithm which will actually compute solutions for arbitrary domains and boundary conditions. A corollary of our results is the non-existence of constructive solutions to the Navier-Stokes equations of fluid flow. But not all the news is bad: we provide reasonable conditions, omitted in the classical theory but easily satisfied, which ensure the computability of solutions.
\end{abstract}

2010 Mathematics Subject Classification 03F60 (primary); 46S30 (secondary)

Keywords: constructive analysis, Dirichlet problem, Brouwerian example, Markov's principle, omniscience principle.

\section{Introduction}

It is a well-known theorem of classical mathematics that the following Dirichlet problem has a (unique) solution:

Problem 1 (The Dirichlet boundary-value problem) Given an open, bounded integrable set $\Omega$ in $\mathbf{R}^{n}$ and a uniformly continuous boundary condition $f: \partial \Omega \rightarrow \mathbf{R}$, find a function $u$ such that

$$
\Delta u=0 \text { on } \Omega, u(x)=f \text { for all } x \in \partial \Omega .
$$

Less well-known is the fact that, despite there being existence proofs of solutions which at first sight appear to be algorithmic, the theorem has no proof which is entirely constructive-in other words, there is no algorithm which will compute solutions to the Dirichlet problem for arbitrary open, bounded, integrable domains with arbitrary uniformly continuous boundary data. Accordingly, since Dirichlet boundary value problems are a special case of the Navier-Stokes equations of fluid flow, there is no 
universal algorithm that will deliver solutions for arbitrary Navier-Stokes systems. ${ }^{1}$ Working within the Bishop-style constructive mathematical framework (BISH), ${ }^{2}$ we prove this rigorously in Theorem 4 and Proposition 9.

It is possible, however, to recover a lot of the classical theory while respecting computability. We also present the following positive (and perhaps more important) result, formalised in Theorem 7: if the Dirichlet problem has computable weak solutions on arbitrary close internal approximations to a domain $\Omega \subset \mathbf{R}^{N}$ which satisfies some mild regularity conditions, then it has a (perforce unique) computable weak solution on $\Omega$ as well.

Throughout, we use the unqualified term 'existence' to mean constructive (or computable) existence. We assume that the reader has access to general sources on constructive analysis, such as the pioneering reference [3] and its second incarnation [4], or the more recent presentation in [5].

\section{Geometric and measure-theoretic background}

When discussing a boundary-value problem in $\mathbf{R}^{n}$ for $n \geq 2$, we need to take into account the geometric and measure-theoretic properties of the domain. Accordingly, we present some notions defined in [9] and [6]. First, though, we mention that a subset $S$ of a metric space $X$ is located if the distance

$$
\rho(x, S) \equiv \inf \{\rho(x, y): y \in S\}
$$

exists (i.e. is computable) for each $x$ in $X .^{3}$ Every totally bounded subset of $X$ is located. Even if $S$ is not necessarily located, we write

- $\rho(x, S)<r$ to mean that there exists $s \in S$ with $\rho(x, s)<r$,

- $\rho(x, S) \geq r$ to mean that $\rho(x, S) \geq r$ for all $x \in X$, and

- $\rho(x, S)>r$ to mean that $\rho(x, S) \geq r^{\prime}$ for some $r^{\prime}>r$.

Let $S, \Omega$ be subsets of $X$. If there exists $r>0$ such that $x \in \Omega$ whenever $\rho(x, S)<r$, then we say that $S$ is well contained in $\Omega$, and we write $S \subset \subset \Omega$. We define $\Omega$ to be

\footnotetext{
${ }^{1}$ The problem of proving the existence of solutions to the Navier-Stokes equations is one of the so-called Millennium Prize Problems posed by the Clay Mathematics Institute; see [13].

${ }^{2}$ For all practical analytic purposes, BISH can be regarded as mathematics developed with intuitionistic logic and a corresponding set- or type-theoretic framework, such as those in $[1,16]$.

${ }^{3}$ The locatedness of every inhabited subset of $\mathbf{R}$ implies the law of excluded middle; hence not every subset of $\mathbf{R}$ is constructively located.
} 
- edge coherent if $x \in \Omega$ whenever $x \in \bar{\Omega}$ and $\rho(x, \partial \Omega)>0$;

- approximated internally by compact sets if for each $\varepsilon>0$ there exists a compact-that is, complete and totally bounded-set $K \subset \subset \Omega$ such that if $x \in \Omega-K$, then $\rho(x, \partial \Omega)<\varepsilon$.

In keeping with the literature we adopt the usual conventions concerning the notation $\partial \Omega, C^{n}(\Omega), C_{0}^{n}(\Omega), H^{1}(\Omega)$ and $H_{0}^{1}(\Omega): C^{n}(\Omega)$ is the space of real-valued functions $n$ times uniformly differentiable on compact subsets of $\Omega ; C_{0}^{n}(\Omega)$ is the space consisting of elements of $C^{n}(\Omega)$ with compact support well contained in $\Omega ; H^{1}(\Omega)$ is the space of square-summable functions whose weak derivatives are also in $L_{2}(\Omega)$; and $H_{0}^{1}(\Omega)$ is the completion of $C_{0}^{1}(\Omega)$ in $H^{1}(\Omega)$.

In [6], Proposition 23 shows that if $\Omega$ is approximated internally by located sets, then it is edge coherent; and Proposition 24 says that if $\Omega$ is edge coherent, totally bounded, and has totally bounded boundary, then it is approximated internally by compact sets.

An important property applicable to an open subset of $\mathbf{R}^{N}$ is the pointwise exterior cone condition: for each $x \in \partial \Omega$, there exist $r, \theta>0$ and a right circular cone $C$ with vertex $x$, vertex angle $\theta$, and height $r$ such that $C \cap \bar{\Omega}=\{x\}$. To pass from this to the (uniform) exterior cone condition, we need the numbers $r, \theta$ to be constructed independent of the point $x \in \partial \Omega$. Proposition 3.20 of [18] shows that if $\Omega \subset \mathbf{R}^{N}$ is edge coherent, totally bounded, and satisfies the uniform exterior cone condition, then $\Omega$ is approximated internally by compact sets.

The details of constructive integration theory are presented in [4] (Chapter 6), to which we refer the reader. However, it is convenient to present some aspects of that theory here.

Let $X$ be a locally compact metric space, $\mu$ a positive measure on $X$, and $L_{1}(\mu)$ the space of $\mu$-integrable functions on $X$. By a complemented set in $X$ we mean an ordered pair $\mathbf{S}=\left(S^{1}, S^{0}\right)$ of subsets of $X$ such that $\rho(x, y)>0$ for all $x \in S^{1}$ and $y \in S^{0}$; if the resulting characteristic function $\chi_{\mathbf{S}}: S^{1} \cup S^{0} \rightarrow\{0,1\}$, defined by

$$
\chi_{\mathbf{S}}(x)= \begin{cases}1 & \text { if } x \in S^{1} \\ 0 & \text { if } x \in S^{0}\end{cases}
$$

is integrable, we call $\mathbf{S}$ an integrable set, with measure $\mu(\mathbf{S}) \equiv \int \chi_{\mathbf{S}}$. We say that the complemented set $\mathbf{S}$ is compact (respectively, closed) if $S^{1}$ is compact (respectively, closed). When $K$ is a compact subset of $X$, we write $\mathbf{K}$ for the complemented set $(K,-K)$, where

$$
-K \equiv\{x \in X: \rho(x, K)>0\}
$$


is the metric complement of $K ;^{4}$ if, moreover, $\mathbf{K}$ is an integrable set, then we call it-or, loosely, $K$ - an integrable compact set in $X$.

We say that a compact set $K \subset X$ is strongly integrable if the following holds:

there exists $c>0$ such that for each $\varepsilon>0$ there exists $\delta>0$ such that $\left\|\int f-c\right\|<\varepsilon$ whenever $f \in C(X), 0 \leq f \leq 1, f(x)=1$ for all $x \in K$, and $f(x)=0$ for all $x$ with $\rho(x, K) \geq \delta$.

In that case, $\mathbf{K}$ is integrable, and $\mu(\mathbf{K})=c$. Here is the fundamental result about approximating integrable sets by strongly integrable ones. (Note that ' $\mathbf{K}<\mathbf{A}$ ' means that $\chi_{\mathbf{K}} \leq \chi_{\mathbf{A}}$ on a full set.)

Theorem 2 Let $\mu$ be a positive measure on a locally compact metric space $X$, and let $\mathbf{A}$ be an integrable set with positive measure. Then for each $\varepsilon>0$, there exists a strongly integrable compact set $K$ such that $\mathbf{K}<\mathbf{A}$ and $\mu(\mathbf{A}-\mathbf{K})<\varepsilon$.

The Dirichlet problem can be re-stated as:

Problem 3 (The Dirichlet problem) Given an open, bounded integrable set $\Omega$ in $\mathbf{R}^{n}$ and $f$ in $L_{2}(\Omega)$, find a function $u$ such that

$$
\Delta u=f \text { on } \Omega, u(x)=0 \text { for all } x \in \partial \Omega,
$$

Under reasonable conditions on $f,(2)$ is equivalent to (1), in the sense that existence of the solution to either version entails the existence of the solution in the other; see [14, p. 131].

By a weak solution to the Dirichlet problem (2) we mean a function $u$ in $H_{0}^{1}(\Omega)$ such that

$$
\langle u, v\rangle_{H_{0}^{1}(\Omega)} \equiv-\int_{\Omega} \nabla u \cdot \nabla v=\int_{\Omega} f v
$$

for all $v \in H_{0}^{1}(\Omega)$.

Before going into more detail, we remind the reader of some important but related issues. First is the (constructive) stability of solutions: Theorem 2 of [9] states that if $u_{f}$ is the weak solution of the Dirichlet problem (2), then for all functions $g$ in $L_{2}(\Omega)$,

$$
\left\|u_{f}-u_{g}\right\|_{H} \leq \gamma\|f-g\|_{2},
$$

\footnotetext{
${ }^{4}$ This should not be confused with the complement

$$
\sim K \equiv\left\{x \in X: \forall_{y \in K}(\rho(x, y)>0)\right\}
$$
}

of $K$. 
where $\gamma$ is the constant in Poincaré's inequality. From this it follows that for a given function $f$ in $L_{2}(\Omega)$ the Dirichlet problem (2) has at most one weak solution.

Next, there is a group of problems which are constructively equivalent to the existence of weak solutions to (2) (for details, see [9, pp. 658-661] and [8, pp. 1159-1160]):

- The total boundedness in the $L_{2}$ norm of the set

$$
S=\left\{v \in C_{0}^{1}(\Omega):\|v\|_{H} \leq 1\right\} .
$$

- The uniform continuity in the double norm on $H_{0}^{1}(\Omega)^{*}$ of the mapping $\lambda_{v} \mapsto \hat{v}(\xi)$ from $S^{*}$ to $\mathbf{R}$. Here $\lambda_{v}$ denotes the bounded linear functional $u \mapsto\langle u, v\rangle$ on $H_{0}^{1}(\Omega)$.

- The locatedness of $H_{0}^{1}(\Omega)$ in $H_{0}^{1}\left(B_{R}\right)$ for each $R>0$ such that $\Omega \subset \subset B_{R}$. Here, by extending each $u \in H_{0}^{1}(\Omega)$ by zero outside $\Omega$, we regard $H_{0}^{1}(\Omega)$ as a subset of $H_{0}^{1}\left(B_{R}\right)$ where $B_{R}=B(0, R) \subset \mathbf{R}^{n}$.

While these have been identified as equivalent problems, none of these have been solved. Thus in the present paper we establish that the constructive solution to any of these problems requires more than the usual classical hypotheses, and provide some quite reasonable constructively sufficient conditions.

\section{The Dirichlet problem, classically}

\section{Associated with Problem 3 is the Dirichlet energy functional}

$$
J(u)=\int_{\Omega}\left(\|\nabla u\|^{2}+2 u f\right) d x .
$$

Dirichlet's principle states that the following two conditions are (classically) equivalent (see e.g. [17, pp. 178-179 and pp. 186-187]).

(i) The function $u$ is a weak solution of the Dirichlet problem (2). That is, $u$ satisfies (3) whenever $v \in H_{0}^{1}(\Omega)$.

(ii) The function $u$ minimizes the Dirichlet energy functional (4) in $H_{0}^{1}(\Omega)$. That is, $J(u) \leq J(v)$ whenever $v \in H_{0}^{1}(\Omega)$.

Minor modifications convert the classical proof of equivalence into a constructive proof. As with the equivalences identified at the end of the previous section, however, the proof remains silent on the question of how to actually compute the function $u$ in $H_{0}^{1}(\Omega)$ which solves (2). Typical classical approaches to finding a $u$ which satisfies either condition proceed along these lines: 
(i) Define a bounded linear functional $\varphi$ on $H_{0}^{1}(\Omega)$ by

$$
\varphi(v):=-\int_{\Omega} v f d x
$$

and apply the Riesz Representation Theorem to find an element $u$ of $H_{0}^{1}(\Omega)$ such that

$$
\phi(v)=\langle u, v\rangle
$$

for all $v$ in $H_{0}^{1}(\Omega)$; then $u$ solves the Dirichlet problem.

(ii) Construct a minimizing sequence for the functional $J$, the infimum of which is guaranteed to exist by the least upper bound principle; then use (weak) sequential compactness to extract a (weakly) convergent subsequence, the limit of which solves the Dirichlet problem.

Constructively, (i) will not guarantee a solution to the Dirichlet problem, since the representability of $\varphi$ is constructively equivalent to its normability. That is, $\varphi$ can be represented only if its norm can be (explicitly) computed. The reason (ii) will not work constructively lies in the application of the least upper bound principle and the sequential compactness argument, as neither of these are provable using only constructive methods. See [8] for details.

\section{Any general method must go constructively wrong}

In this section we prove that the existence of a weak solution of the general Dirichlet problem for a domain $\Omega \subset \mathbf{R}^{2}$ cannot be proved constructively. To make the following examples easier to follow, we return to the version (1) from the introduction.

Still working within BISH, we remind the reader of two important principles: Markov's principle,

MP: For each binary sequence $\left(a_{n}\right)_{n \geq 1}$, if it is impossible that $a_{n}=0$

for all $n$, then there exists $n$ such that $a_{n}=1$,

and the limited principle of omniscience,

LPO: For each binary sequence $\left(a_{n}\right)_{n \geq 1}$, either $a_{n}=0$ for all $n$, or else there exists $n$ such that $a_{n}=1$.

Markov's principle is consistent with (though not included in) BISH, is commonly employed in recursive constructive mathematics (essentially, BISH plus the ChurchMarkov-Turing thesis and Markov's principle—see [15]), but, with the aid of Brouwer's 
theory of the creating subject (see [11]) is provably false in intuitionistic mathematics. On the other hand, LPO (which clearly implies MP) is recursively and intuitionistically false, and is regarded as essentially nonconstructive.

We are aiming for this result:

Theorem 4 The following are equivalent over BISH.

(i) LPO.

(ii) Markov's principle holds, and for every totally bounded, Lebesgue integrable, open subset $\Omega$ of $\mathbf{R}^{N}$, and every uniformly continuous function $f: \partial \Omega \rightarrow \mathbf{R}$, the Dirichlet problem has a weak solution in $H_{0}^{1}(\Omega)$.

Before proving this, though, we put on record a simple, but useful, preliminary proposition, and describe a key construction that we use in the proof:

Proposition 5 LPO $\vdash$ If $S$ is a countable subset of $\mathbf{R}$, then either $S$ is bounded above or else it is unbounded above (that is, for each $c>0$, there exists $x \in S$ with $x>c$ ). Moreover, if $S$ is bounded above, then $\sup S$ exists. ${ }^{5}$

Proof Let $f$ be a mapping of $\mathbf{N}^{+}$onto $S$. Given $\alpha, \beta \in \mathbf{R}$, construct a binary sequence $\lambda \equiv\left(\lambda_{n}\right)_{n \geq 1}$ such that if $\lambda_{n}=0$, then $f(n)<\beta$, and if $\lambda_{n}=1$, then $f(n)>\alpha$. Applying LPO to $\lambda$, we see that either $f(n)<\beta$ for all $n$ or else there exists $n$ such that $f(n)>\alpha$.

Without loss of generality, we may assume that $f(1)>1$. Setting $\mu_{1}=0$, construct a binary sequence $\mu \equiv\left(\mu_{n}\right)_{n \geq 1}$ such that for $n \geq 2$, if $\mu_{n}=0$, then there exists $m$ such that $f(m)>n$, and if $\mu_{n}=1$, then $f(m)<n+1$ for all $m$. Applying LPO to $\mu$, we see that either $S$ is unbounded above or else it is bounded above. In the latter case, noting the first paragraph of this proof, we see from the constructive least-upper-bound principle ([5], Theorem 2.1.18) that $\sup S$ exists.

The following lemma embodies our key construction for the proof of Theorem 4.

Lemma 6 Let $\left(a_{n}\right)_{n \geq 1}$ be an increasing binary sequence with $a_{1}=0$. Let $D$ be the open unit disc in $\mathbf{R}^{2}$, and for each positive integer $n$ define

$$
T_{n} \equiv\left\{x \in \mathbf{R}^{2}: \frac{1}{n}<\|x\|<1\right\} .
$$

\footnotetext{
${ }^{5}$ It follows that, in contrast to the classical least-upper bound principle for arbitrary inhabited, bounded subsets of $\mathbf{R}$, which is equivalent, over BISH, to the full law of excluded middle, the same principle for countable subsets is equivalent to LPO.
} 
If $a_{n}=0$, set $\Omega_{n} \equiv D$; if $a_{n}=1-a_{n-1}$, set $\Omega_{k} \equiv T_{n}$ for all $k \geq n$. Define

$$
\Omega=\left(\bigcap_{n \geq 1} \Omega_{n}\right)^{\circ} \text {. }
$$

Then $\Omega$ is totally bounded, located and integrable.

Proof First we prove that $\Omega$ is totally bounded and hence located. Observe that for each $n, \Omega_{n}$ is totally bounded and $\Omega_{n+1} \subset \Omega_{n}$, and that if $a_{n}=0$, then $T_{n} \subset \Omega$. Given $\varepsilon>0$, compute a positive integer $N>1 / \varepsilon$. If $a_{2 N}=1$, then $\Omega=\Omega_{m}$ for some $m \leq N$, so we can certainly construct a finite $\varepsilon$-approximation to $\Omega$. On the other hand, if $a_{2 N}=0$, then $T_{2 N} \subset \Omega$, and we construct a finite $\varepsilon / 3$-approximation $\left\{x_{1}, \ldots, x_{n}\right\}$ to $T_{2 N}$. Given $x \in \Omega$, we have either $\|x\|>1 / 2 N$ or $\|x\|<1 / N$. In the former case, $x \in T_{2 N}$, so we can choose $k \leq n$ such that $\left\|x-x_{k}\right\|<\varepsilon / 3<\varepsilon$. In the case $\|x\|<1 / N$, we have $\rho\left(x, T_{2 N}\right)<2 / 3 N<2 \varepsilon / 3$; choosing $y \in T_{2 N}$ with $\|x-y\|<2 \varepsilon / 3$, and then $k \leq n$ such that $\left\|y-x_{k}\right\|<\varepsilon / 3$, we obtain $\left\|x-x_{k}\right\|<\varepsilon$. Thus $\left\{x_{1}, \ldots, x_{n}\right\}$ is a finite $\varepsilon$-approximation to $\Omega$. Since $\varepsilon>0$ is arbitrary, we conclude that $\Omega$ is totally bounded (and thus located).

To show that $\Omega$ is integrable, first observe that for each $n, \Omega_{n}$ is integrable. Construct the sequence of real numbers $\left(I_{n}\right)_{n \geq 1}$ such that $I_{n}=\int \chi_{\Omega_{n}}$ for each $n$. Now for $m \geq n$,

$$
\left\|I_{n}-I_{m}\right\|=\left\|\int \chi_{\Omega_{m}}-\int \chi_{\Omega_{n}}\right\| \leq \frac{\pi}{n^{2}}
$$

and so $\left(I_{n}\right)$ is a Cauchy sequence and converges to a limit $I$. A corollary of the constructive Lebesgue series theorem (a consequence of $[4, \mathrm{Ch} .6(2.17)]$ ) now informs us that $\Omega$ is integrable and has measure $\lim _{n \rightarrow \infty} \int \chi_{\Omega_{n}}$.

Figure 1 illustrates this construction.

Turning back to the Dirichlet problem, we give the proof of Theorem 4:

Proof First assume $\mathbf{L P O}$ and consider the Dirichlet problem (1) with $\Omega \subset \mathbf{R}^{N}$ totally bounded, Lebesgue integrable, and open, and with $f: \partial \Omega \rightarrow \mathbf{R}$ uniformly continuous. Since LPO implies MP, it will suffice to prove that this Dirichlet problem has a weak solution; we base the argument on the Ritz-Galerkin method (see Chapter 9 of [2]). Let $\left(e_{n}\right)_{n \geq 1}$ be an orthonormal basis of $H_{0}^{1}(\Omega)$, and for each $n$ let $H_{n}$ be the finitedimensional subspace of $H_{0}^{1}(\Omega)$ spanned by $\left\{e_{1}, \ldots, e_{n}\right\}$. Define a linear functional $\phi$ on $H_{0}^{1}(\Omega)$ by

$$
\phi(v) \equiv-\int_{\Omega} v f
$$



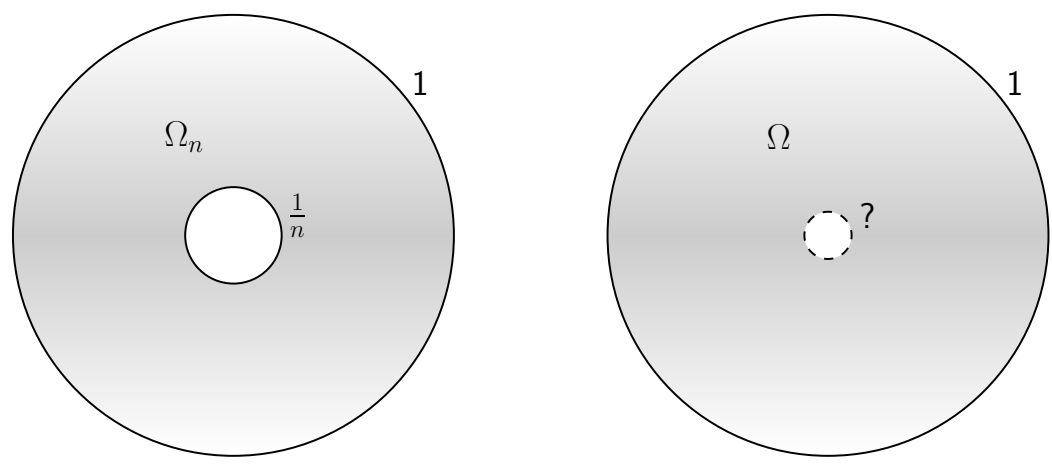

Figure 1: The domain $\Omega_{n}$ (left) when $a_{n}=1-a_{n-1}$, used in the construction from Lemma 6; and the domain $\Omega$ (right), formed by intersecting the $\Omega_{n}$.

Standard estimates, using the Hölder and Poincaré inequalities [12] (Chapter 5, Theorem 3), yield

$$
\|\phi(v)\| \leq \gamma\|f\|_{2}\|v\|_{H_{0}^{1}(\Omega)} \quad\left(v \in H_{0}^{1}(\Omega)\right),
$$

where $\gamma$ is the constant in Poincaré's inequality. Thus the linear functional $\phi$ is bounded. For each $n$, since $H_{n}$ is finite-dimensional, the restriction of $\phi$ to $H_{n}$ is normed: ${ }^{6}$ that is,

$$
\left\|\left.\phi\right|_{H_{n}}\right\| \equiv \sup \left\{\|\phi(v)\|: v \in H_{n}\right\}
$$

exists; moreover (recall (5))

$$
\left\|\left.\phi\right|_{H_{n}}\right\| \leq\left\|\left.\phi\right|_{H_{n+1}}\right\| \leq \gamma\|f\|_{2} .
$$

It follows from this and Proposition 5 that

$$
\sigma \equiv \sup \left\{\left\|\left.\phi\right|_{H_{n}}\right\|: n \geq 1\right\}=\lim _{n \rightarrow \infty}\left\|\left.\phi\right|_{H_{n}}\right\|
$$

exists. We show that $\phi$ is normed, with $\|\phi\|=\sigma$. Let $P_{n}$ be the projection of $H_{0}^{1}(\Omega)$ onto $H_{n}$. For each $v \in H_{0}^{1}(\Omega)$,

$$
\phi(v)=\lim _{n \rightarrow \infty} \phi\left(P_{n} v\right) \leq \lim _{n \rightarrow \infty}\left\|\left.\phi\right|_{H_{n}}\right\|\|v\|_{H_{0}^{1}(\Omega)}=\sigma\|v\|_{H_{0}^{1}(\Omega)} .
$$

On the other hand, given $\varepsilon>0$ and choosing $n$ such that $0 \leq \sigma-\left\|\left.\phi\right|_{H_{n}}\right\|<\varepsilon$, we can find a unit vector $v \in H_{n}$ such that

$$
\left\|\left.\phi\right|_{H_{n}}\right\|-\varepsilon<\|\phi(v)\| \leq\left\|\left.\phi\right|_{H_{n}}\right\| \text {. }
$$

\footnotetext{
${ }^{6}$ The statement every bounded linear functional on an infinite-dimensional Hilbert space is normed implies LPO. The Riesz representation theorem for linear functionals applies if and only if the functional is not just bounded, but normed ([5], Theorem 4.3.6).
} 
Then

$$
0 \leq \sigma-\|\phi(v)\|=\sigma-\left\|\left.\phi\right|_{H_{n}}\right\|+\left\|\left.\phi\right|_{H_{n}}\right\|-\|\phi(v)\|<2 \varepsilon .
$$

Since $\varepsilon>0$ is arbitrary, we conclude that $\|\phi\|$ exists and equals $\sigma$. We are now able to apply the (constructive) Riesz representation theorem ([5], Theorem 4.3.6), to produce $w \in H_{0}^{1}(\Omega)$ such that for each $v \in H_{0}^{1}(\Omega)$,

$$
-\int_{\Omega} v f=\phi(v)=\langle v, w\rangle_{H_{0}^{1}(\Omega)} .
$$

The function $w$ is therefore the sought-after weak solution of the Dirichlet problem. Hence (i) implies (ii).

Suppose, conversely, that (ii) holds, and consider the Dirichlet problem

$$
\Delta u=0 \text { on } \Omega, u(x)=\log \|x\| \text { for each } x \in \partial \Omega .
$$

where $\Omega$ is the domain from Lemma 6. Since $(0,0) \notin \partial \Omega$ and we are assuming Markov's principle, for each $x \in \partial \Omega$ we have $\|x\|>0$ and therefore $\log \|x\|$ welldefined. We show that the function $f: x \rightsquigarrow \log \|x\|$ is uniformly continuous on $\partial \Omega$. Let $x, x^{\prime} \in \partial \Omega$ be such that $\left\|x-x^{\prime}\right\|<1 / 2$. Either $\min \left\{\|x\|,\left\|x^{\prime}\right\|\right\}>1 / 2$ or $\min \left\{\|x\|,\left\|x^{\prime}\right\|\right\}<1$. In the first case we must have $\|x\|=\left\|x^{\prime}\right\|=1$, so $\log \|x\|=$ $\log \left\|x^{\prime}\right\|=0$. In the case $\min \left\{\|x\|,\left\|x^{\prime}\right\|\right\}<1$, we may assume that $\|x\|<1$; since (as we observed above) $\|x\|>0$, there exists $N>2$ such that $\Omega=\Omega_{N},\|x\|=1 / N$, and therefore $\left\|x^{\prime}\right\|<\|x\|+1 / 2<1$. Hence $\left\|x^{\prime}\right\|=1 / N=\|x\|$. Thus if $x, x^{\prime} \in \partial \Omega$ and $\left\|x-x^{\prime}\right\|<1 / 2$, then $f(x)=f\left(x^{\prime}\right)$.

If $a_{n}=0$ for all $n$, then our Dirichlet problem has the unique (weak and strong) solution $u(x)=0$ for all $x \in \bar{\Omega}$, and $\int_{\Omega} u^{2}=0$. If $a_{n}=1-a_{n-1}$ for some $n$, then (6) has the unique (weak and strong) solution $u(x)=\log \|x\|$ for all $x \in \bar{\Omega}$, and, with the usual polar coordinates $r$ and $\theta$,

$$
\begin{aligned}
\int_{\Omega} u^{2} & =\iint_{\Omega} r(\log r)^{2} \mathrm{~d} r \mathrm{~d} \theta \\
& =\pi\left(\frac{1}{2}-\frac{1}{n^{2}} \log ^{2} \frac{1}{n}+\frac{1}{n^{2}} \log \frac{1}{n}-\frac{1}{2 n^{2}}\right) .
\end{aligned}
$$

Moreover, the last expression is an increasing function of $n$ that converges to $\pi / 2$ as $n \rightarrow \infty$, and in the case $n=2$ is larger than $1 / 4$. Assume that the Dirichlet problem (6) has a weak solution $w \in L_{2}(\bar{\Omega})$. Then either $\int_{\Omega} w<1 / 4$ or $\int_{\Omega} w>0$. In the first case, we must have $a_{n}=0$ for all $n$; in the second, it is impossible that $a_{n}=0$ for all $n$, so, by Markov's principle, there exists $n$ such that $a_{n}=1$. This completes the proof that (ii) implies LPO. 


\section{Constructing a weak solution}

In this section we examine the Dirichlet problem. Specifically, in this section we provide conditions that ensure the existence of a weak solution of (2): that is, an element $u$ of the Hilbert space $H_{0}^{1}(\Omega)$ such that ${ }^{7}$

$$
\langle u, v\rangle_{H_{0}^{1}(\Omega)} \equiv-\int_{\Omega} \nabla u \cdot \nabla v=\int_{\Omega} f v \quad\left(v \in C_{0}^{1}(\Omega)\right) .
$$

We call a subset $\Omega$ of $\mathbf{R}^{N}$ a Wang domain if it has the following properties:

(i) It is edge coherent, totally bounded, and open in $\mathbf{R}^{N}$.

(ii) $\partial \Omega$ is compact.

(iii) The complemented set $\Omega \equiv(\Omega,-\Omega)$, is Lebesgue integrable, and the divergence theorem applies to it and $\partial \Omega$.

(iv) There exists $c_{0}>0$ such that if

$$
(\partial \Omega)_{r} \equiv\{x \in \bar{\Omega}: \rho(x, \partial \Omega) \leq r\}
$$

is integrable, then for each $u$ in

$$
\int_{(\partial \Omega)_{r}}\|u\|^{2} \leq c_{0} r^{2} \int_{(\partial \Omega)_{r}}\|\nabla u\|^{2} \quad\left(u \in H_{0}^{1}(\Omega)\right) .
$$

Notice that conditions (i)-(iii) are classically trivial, in the sense that, using classical logic, any bounded domain is easily seen to satisfy them. Yet they yield important computational information: (i) and (ii) embody information about the boundary (classically taken for granted), and (iii) holds as a consequence of the (classical) divergence theorem. Condition (iv) is an inequality which provides information about the norm of candidates for the solution (elements of $H_{0}^{1}(\Omega)$ ); it asserts that the average value of $\|u\|^{2}$ in a band of width $r$ about $\partial \Omega$ tends to zero with $r$ and is motivated by the classical theory (see e.g. [17, p. 199, Theorem 6]). These conditions are easily seen to hold classically for domains with located boundary, so examples abound: the unit ball in $\mathbf{R}^{n}$ is the canonical example.

Theorem 7 Let $\Omega$ be a Wang domain in $\mathbf{R}^{N}$, and let $f \in L_{2}(\Omega)$ (relative to Lebesgue measure $\mu$ on $\Omega$ ). Suppose that there exists a sequence $\left(\Omega_{n}\right)_{n \geq 1}$ of edge coherent, totally bounded, open subsets of $\Omega$, each having compact boundary, such that for each $n$,

\footnotetext{
${ }^{7}$ We assume that the reader is familiar with, or has access to, the definition and basic properties of the spaces $H_{0}^{1}(\Omega)$ and $H_{0}^{2}(\Omega)$, as found in [12] or [17].
} 
$\triangleright \overline{\Omega_{n}} \subset \subset \Omega_{n+1}$,

$\triangleright \Omega_{n}$ and $\partial \Omega_{n}$ are Lebesgue integrable, with $\mu\left(\partial \Omega_{n}\right)=0$,

$\triangleright \max \left\{\rho\left(\overline{\Omega_{n}}, \bar{\Omega}\right), \rho\left(\partial \Omega_{n}, \partial \Omega\right)\right\} \rightarrow 0$ as $n \rightarrow \infty$, and

$\triangleright$ the Dirichlet problem

$$
\Delta u=f \text { on } \Omega_{n}, u(x)=0 \text { for all } x \in \partial \Omega_{n}
$$

has a weak solution $u_{n}$ in $H_{0}^{1}\left(\Omega_{n}\right)$.

Then the sequence $\left(u_{n}\right)_{n \geq 1}$ converges in $H_{0}^{1}(\Omega)$ to a weak solution of the Dirichlet problem (1)).

Proof According to [7] (or Lemma 6 of [9]), there exists $\kappa>0$ such that if $K \subset \mathbf{R}^{N}$ is a compact set and $\varepsilon>0$, then there exists a $C^{\infty}$ cutoff function $\eta: \mathbf{R}^{N} \rightarrow[0,1]$ such that $\eta(x)=1$ for all $x \in K, \eta(x)=0$ whenever $\rho(x, K) \geq \varepsilon$, and $\|\nabla \eta(x)\| \leq \kappa / \varepsilon$ for all $x \in \mathbf{R}^{N}$. For convenience, if $r>0$, we write

$$
K_{r} \equiv\left\{x \in \mathbf{R}^{N}: \rho(x, K) \leq r\right\} .
$$

Fix $\varepsilon>0$, and choose $t>0$ such that if $S$ is an integrable set with $\mu(\Omega-S)<t$, then

$$
\int_{\Omega-S}\|\nabla u\|^{2}<\frac{2 \varepsilon^{2}}{9\left(1+25 c_{0} \kappa^{2}\right)} .
$$

As in the proof of Theorem 3 of [9], we can find $\delta>0$ such that

$$
K \equiv\{x \in \bar{\Omega}: \rho(x, \sim \Omega) \geq 5 \delta\}
$$

is compact and integrable, $\mu(\Omega-K)<t$, and $K_{2 \delta} \subset \subset \Omega_{n}$ whenever

$$
\max \left\{\rho\left(\overline{\Omega_{n}}, \bar{\Omega}\right), \rho\left(\partial \Omega_{n}, \partial \Omega\right)\right\}<\delta .
$$

Construct a $C^{\infty}$ function $\alpha: \mathbf{R}^{N} \rightarrow[0,1]$ such that $\alpha(x)=0$ for all $x \in K, \alpha(x)=1$ for all $x \in-K_{\delta}$, and $\|\nabla \alpha(x)\| \leq \kappa / \delta$ for all $x \in \mathbf{R}^{N}$. Then compute $N$ such that

$$
\max \left\{\rho\left(\overline{\Omega_{n}}, \bar{\Omega}\right), \rho\left(\partial \Omega_{n}, \partial \Omega\right)\right\}<\delta \quad(n \geq N) .
$$

Given $n \geq N$, set $w \equiv u_{n}-u_{N}$. Then, by similar arguments to ones in the proof of Theorem 3 of [9],

$$
\int_{\Omega_{N}} \nabla w \cdot \nabla\left(w-\alpha u_{n}\right)=0
$$

and hence

$$
4 \int_{\Omega_{N}}\|\nabla w\|^{2} \leq 9 \int_{\Omega_{n}-K}\|\nabla u\|^{2}+\frac{9 \kappa^{2}}{\delta^{2}} \int_{\Omega_{n}-K}\left\|u_{n}\right\|^{2} .
$$


We also get

$$
\Omega_{n}-K \subset \bar{\Omega}-K \subset \bar{\Omega} \cap K_{5 \delta} .
$$

Since $\mu\left(\partial \Omega_{n}\right)=0$ and $u=0$ on $-\Omega_{n}$, we see from (7) that

$$
\int_{\Omega_{n}-K}\left\|u_{n}\right\|^{2}=\int_{(\partial \Omega)_{5 \delta}}\left\|u_{n}\right\|^{2} \leq 25 c_{0} \delta^{2} \int_{\Omega-K}\|\nabla u\|^{2} .
$$

Hence

$$
4 \int_{\Omega_{N}}\left\|\nabla u_{n}-u_{N}\right\|^{2}=4 \int_{\Omega_{N}}\|\nabla w\|^{2} \leq 9\left(1+25 c_{0} \kappa^{2}\right) \int_{\Omega-K}\|\nabla u\|^{2} .
$$

Since $\mu\left(\Omega_{n}-K\right)<t$, it follows that

$$
\int_{\Omega_{N}}\left\|\nabla u_{n}-\nabla u_{N}\right\|^{2}<\frac{\varepsilon^{2}}{2} .
$$

Also, $K \subset \Omega_{N}$ and therefore $\mu(\Omega-K)<t$; whence

$$
\begin{aligned}
\int_{\Omega}\left\|\nabla u_{n}-\nabla u_{N}\right\|^{2} & =\int_{\Omega-\Omega_{N}}\|\nabla u\|^{2}+\int_{\Omega_{N}}\left\|\nabla u_{n}-\nabla u_{N}\right\|^{2} \\
& <\frac{\varepsilon^{2}}{2}+\frac{\varepsilon^{2}}{2}=\varepsilon^{2}
\end{aligned}
$$

and therefore $\left\|u_{n}-u_{N}\right\|_{H_{0}^{1}(\Omega)}<\varepsilon$.

We now see that $\left\|u_{m}-u_{n}\right\|_{H_{0}^{1}(\Omega)}<2 \varepsilon$ whenever $m \geq n \geq N$. Thus $\left(u_{n}\right)_{n \geq 1}$ is a Cauchy sequence in the complete space $H_{0}^{1}(\Omega)$. Let $u$ be its limit. Given $v \in C_{0}^{1}(\Omega)$, we compute

$$
\begin{aligned}
\int_{\Omega} \nabla u_{n} \cdot \nabla v & =\int_{\Omega_{n}} \nabla u_{n} \cdot \nabla v+\int_{\Omega-\Omega_{n}} \nabla u_{n} \cdot \nabla v \\
& =-\int_{\Omega_{n}} f v+\int_{\Omega-\Omega_{n}} \nabla u_{n} \cdot \nabla v \\
& =-\int_{\Omega} f v+\int_{\Omega-\Omega_{n}} f v+\int_{\Omega-\Omega_{n}} \nabla u_{n} \cdot \nabla v .
\end{aligned}
$$

Let $L$ be a compact support of $v$ well-contained in $\Omega$. Compute $n_{1}$ such that $L \subset \subset \Omega_{n_{1}}$. Then $v=\nabla v=0$ throughout $\Omega-\Omega_{n_{1}}$, so

$$
\int_{\Omega-\Omega_{n}} f v=\int_{\Omega-\Omega_{n}} \nabla u_{n} \cdot \nabla v=0 \quad\left(n \geq n_{1}\right) .
$$

Thus

$$
\langle u, v\rangle_{H_{0}^{1}(\Omega)}=\lim _{n \rightarrow \infty}\left\langle u_{n}, v\right\rangle=\lim _{n \rightarrow \infty} \int_{\Omega} \nabla u_{n} \cdot \nabla v=-\int_{\Omega} f v .
$$

Since $v \in C_{0}^{1}(\Omega)$ is arbitrary, we conclude that $u$ is the weak solution of the Dirichlet problem (1). 
Typical domains involving applications (such as the unit ball, unit cube, etc.) often satisfy the conditions of Theorem 7. The paper [10] shows that Green functions can be effectively constructed for a large class of domains, and hence on such domains the Dirichlet problem admits a constructive solution.

We now turn to some properties of the counterexample presented in Lemma 6.

\section{Revisiting the Brouwerian example in Lemma 6}

Recall that we already have established the locatedness and integrability of the set $\Omega$ constructed in Lemma 6. We now outline proofs of some properties of this domain.

(a) The domain $\Omega$ satisfies a pointwise exterior cone condition, but if it satisfies a uniform one, then

$$
\forall_{n}\left(a_{n}=0\right) \vee \neg \forall_{n}\left(a_{n}=0\right)
$$

Since for each $x \in \partial \Omega$, either $\|x\|>1 / 2$ or $0<\|x\|<1$, we easily show that $\Omega$ satisfies the pointwise exterior cone condition. But if $\Omega$ satisfies the uniform exterior cone condition, then LPO holds. To see this, pick $r, \theta>0$ such that for each $x \in \partial \Omega$, there exists a right circular cone with vertex $x$, vertex angle $\theta$, and height $r$ such that $C \cap \bar{\Omega}=\{x\}$; then compute a positive integer $N>1 / 2 r$. Then it is impossible that $a_{n}=1-a_{n-1}$ for some $n \geq N$, so if $a_{N}=0$, then $a_{n}=0$ for all $n$.

It should be noted that classically the domain $\Omega$ does satisfy the exterior cone condition.

(b) The domain $\Omega$ is edge coherent.

To see this, first note that $\Omega_{n}$ is edge coherent for each $n$. Given $x \in \bar{\Omega}$ with $\rho(x, \partial \Omega)>0$, pick $r$ such that $0<r<\min \left(\frac{1}{4}, \rho(x, \partial \Omega)\right)$. Either $x \neq 0$ or $\|x\|<r / 2$. In the first case, pick a positive integer $N>1 /\|x\|$. If $a_{N}=0$, then $x \in \Omega_{N} \subset \Omega$. If $a_{N}=1$, then there exists $m \leq N$ such that $x \in \Omega_{m}=\Omega$. In the case $\|x\|<r$, for each $y \in \partial \Omega$ we have $\|y\| \geq\|x-y\|-\|x\|>r / 2$; whence $\rho(0, \partial \Omega) \geq r / 2$. Computing an integer $m>2 / r$, we see that if $a_{n}=1-a_{n-1}$ for some $n \geq m$, then $\Omega=\Omega_{n}$ and $\rho(0, \partial \Omega)=1 / n<r / 2$, a contradiction. It follows that if $a_{m}=0$, then $a_{n}=0$ for all $n$; whence $\Omega=D$, and so $x \in \bar{\Omega}$. On the other hand, if $a_{m}=1$, then $\Omega=\Omega_{n}$ for some $n \leq m$, again $\Omega$ is edge coherent, and so $x \in \bar{\Omega}$.

(c) If $\Omega$ is approximated internally by compact sets then (9) holds. 
Suppose that $\Omega$ is approximated internally by compact sets. Choose a compact set $K \subset \subset \Omega$ such that if $x \in \Omega-K$, then $\rho(x, \partial \Omega)<1 / 4$, and compute $r>0$ such that if $\rho(x, K) \leq r$, then $x \in \Omega$. Either $r>\delta \equiv \rho((0,0), K)$, in which case $(0,0) \in \Omega$ and we must have $a_{n}=0$ for all $n$, or else, as we suppose, $\delta>0$. Now pick $y \in K$ such that $\|y\|<\delta+r$, and set

$$
z \equiv \frac{\delta}{\delta+r} y
$$

Then $\|z\|<\delta$, so $\rho(z, K)>0$; also,

$$
\rho(z, K) \leq\|z-y\|<r
$$

so $z \in \Omega$. It follows that $\rho(z, \partial \Omega)<1 / 4$. Now choose $w \in \partial \Omega$ such that $\|z-w\|<$ $1 / 4$. If $\|w\|<1$, then it is impossible that $a_{n}=0$ for all $n$. If $\|w\|>3 / 4$, then $\|z\|>1 / 2$ and therefore $\delta>1 / 2$. Suppose that $a_{N}=1-a_{N-1}$ for some $N>4$; then $\Omega=\Omega_{N}$. Since $\delta>1 / 2$, we can find $\zeta \in-K$ with $1 / 2<\|\zeta\|<3 / 4$. Then $\zeta \in \Omega-K$, so there exists $v \in \partial \Omega$ with $\|\zeta-v\|<1 / 4$ and therefore $\|v\|<1$. Thus $\|v\|=1 / N<1 / 4$, which is absurd since $\|\zeta\|>1 / 2$. We conclude from this contradiction that $a_{n}=a_{4}$ for all $n \geq 4$. It follows that in both our cases, either $a_{n}=0$ for all $n$ or it is impossible that $a_{n}=0$ for all $n$.

(d) If $\partial \Omega$ is totally bounded, then (9) holds.

We have already remarked, in the introduction, that if $\Omega$ is edge coherent and totally bounded, and has totally bounded boundary, then it is approximated internally by compact sets. It follows from (b) and (c) that if $\partial \Omega$ is totally bounded, then either $a_{n}=0$ for all $n$ or else it is impossible that $a_{n}=0$ for all $n$.

(e) Although $\Omega$ is located, if $\partial \Omega$ is located, then (9) holds.

Indeed, suppose that $\partial \Omega$ is located; then either $\rho\left(\left(\frac{1}{4}, 0\right), \partial \Omega\right)>1 / 4$, in which case $a_{n}=0$ for all $n$, or else $\rho\left(\left(\frac{1}{4}, 0\right), \partial \Omega\right)<1 / 2$, in which case it is impossible that $a_{n}=0$ for all $n$.

(f) If

$$
(\partial \Omega)_{r} \equiv\{x \in \bar{\Omega}: \rho(x, \partial \Omega) \leq r\}
$$

is integrable, then (9) holds.

If $(\partial \Omega)_{r}$ is Lebesgue integrable then since $\left\{x \in \mathbf{R}^{2}:\|x\|=1\right\} \subset \partial \Omega,(\partial \Omega)_{r}$ has positive measure. Choose $r$ and $\varepsilon$ such that $0<r \leq 1 / 8$ and $\varepsilon<\pi r^{2} / 4$. By Theorem 2 , there exists an integrable compact set $K \subset(\partial \Omega)_{r}$ such that

$$
\mu\left((\partial \Omega)_{r}-K\right)<\varepsilon .
$$


As $K$ is compact, it is located. First, observe that the unit circle is in $\partial \Omega$ (and the measure of that component of $(\partial \Omega)_{r}$ is $\left.4 \pi r\right)$; call this component of $\partial \Omega$ the outer component. Call any other component of $\partial \Omega$, if there is one, the inner component.

Now either $\rho(0, K)>r$ or $\rho(0, K)<2 r$. If $\rho(0, K)>r$, then there exists a positive integer $N$ such that $1 / r<N<2 / r$. Suppose that there exists some $n \geq N$ such that $a_{n}=a_{n-1}-1$. Then there is an inner component of $\partial \Omega$ closer to 0 than $1 / N$. The measure of the corresponding inner component of $(\partial \Omega)_{r}$ is at least $\pi r^{2}$, and so by (10) and our choice of $\varepsilon$ there must be a corresponding inner component of $K$. Since $N>1 / r$ and $a_{n}=0$ for all $n<N$, we have

$$
\left\{x:\|x\|<\frac{1}{N}\right\} \subset(\partial \Omega)_{r} .
$$

Since $N<2 / r$,

$$
\mu\left(\left\{x:\|x\|<\frac{1}{N}\right\}\right)=\frac{\pi}{N^{2}}>\frac{\pi r^{2}}{4}
$$

and so $\rho(0, K) \leq 1 / N<r$, a contradiction; hence if $a_{n}=0$ for each $n \leq N$, then $a_{n}=0$ for all $n$. On the other hand, if $\rho(0, K)<2 r \leq 1 / 4$, then it is impossible that $a_{n}=0$ for all $n$.

We see from comments (a) and (c)-(f) that in each case, if a certain property holds no matter what binary sequence $\left(a_{n}\right)_{n \geq 1}$ is used to define $\Omega$, then we can derive the following:

WLPO: For each binary sequence $\left(a_{n}\right)_{n \geq 1}$, either $a_{n}=0$ for all $n$ or it is impossible that $a_{n}=0$ for all $n$.

a lesser version of LPO. We can move from WLPO to the stronger omniscience property LPO in these situations if we allow ourselves the use of Markov's principle; since we made use of MP in the proof of Theorem 4, its further application here is not entirely without warrant. However, forgoing MP in (a) and (c)-(f) shows that each of the properties of $\Omega$ in question is genuinely nonconstructive.

(g) Assuming Markov's principle, we may compute a compact subset $K_{\infty}$ of $\bar{\Omega}$ such that

$$
\partial \Omega=\{x:\|x\|=1\} \cup\left(K_{\infty}-\{0\}\right) .
$$

Moreover, $K_{\infty}-\{0\}$ is inhabited if and only if $a_{n}=1$ for some $n$.

To prove this, first observe that

$$
\partial \Omega=\{x:\|x\|=1\} \cup\left\{x: \exists_{n}\left(\|x\|=n^{-1} \wedge a_{n}=1-a_{n-1}\right)\right\} .
$$


If $a_{n}=0$, set $K_{n}=\{0\}$; if $a_{n}=1-a_{n-1}$, set

$$
K_{j}=\left\{x:\|x\|=n^{-1}\right\} \quad(j \geq n) .
$$

Then each $K_{n}$ is compact, and $\rho\left(K_{m}, K_{n}\right) \leq n^{-1}$ whenever $m \geq n$, where $\rho$ is the Hausdorff metric. Since the set of all compact subsets of the compact space $D$ is complete relative to the Hausdorff metric ([3], page 109, Exercise 12), there exists a compact set $K_{\infty} \subset D$ such that $\rho\left(K_{n}, K_{\infty}\right) \rightarrow 0$ as $n \rightarrow \infty$. In fact, we have $\rho\left(K_{\infty}, K_{n}\right) \leq n^{-1}$ for each $n$. Now, given $x \in \partial \Omega$ and assuming Markov's principle, we have $x \neq 0$ and so we can pick a positive integer $N>\|x\|^{-1}$. If $a_{N}=0$, then $\|x\|=1$; if $a_{N}=1$, then there exists $\nu \leq N$ such that $a_{\nu}=1-a_{\nu-1}, K_{\infty}-\{0\}=K_{\nu}$, and $\|x\|=\nu^{-1}$; whence $x \in K_{\infty}-\{0\}$. Conversely, if $\|x\|=1$, then $x \in \partial \Omega$; whereas if $x \in K_{\infty}-\{0\}$, then we easily show that there exists $n$ with $K_{\infty}=K_{n} \subset \partial \Omega$. This completes the proof of (11). It is a simple exercise to prove that $K_{\infty}-\{0\}$ is inhabited if and only if there exists $n$ with $a_{n}=1$.

(h) The divergence theorem, suitably tweaked, holds for uniformly continuous functions on $\bar{\Omega}$.

In order to make sense of the divergence theorem in the context of the proof that (ii) implies (i) in Theorem 4, we need to be able to construct the appropriate measure for integrals over $\partial \Omega$. Here we have a clear difficulty, since measures are defined for locally compact spaces, and local compactness for $\partial \Omega$ is equivalent to locatedness, which runs afoul of (d) above. However, we can still act as if we knew the exact position of $\partial \Omega$, as follows. Let $u: \bar{\Omega} \rightarrow \mathbf{R}$ be uniformly continuous and let $\|u\| \leq c$, where $c>0$. If $a_{n}=0$, define $I_{n} \equiv 0$. If $a_{n}=1-a_{n-1}$, then, referring to (f) above, we see that $K_{\infty}=K_{n}$, and we define

$$
I_{m} \equiv \frac{1}{n} \int_{\|x\|=1 / n} u(x) \mathrm{d} \theta(x) \quad(m \geq n),
$$

where $\theta(x)$ is polar measure on the circle. We obtain the inequality $\left\|I_{m}-I_{n}\right\| \leq 2 \pi c / n$ for all $m \geq n$; whence $\left(I_{n}\right)_{n \geq 1}$ is a Cauchy sequence and therefore converges to a limit $I_{\infty} \in \mathbf{R}$. If we define

$$
\int_{\partial \Omega} u \equiv \int_{\|x\|=1} u(x) \mathrm{d} \theta(x)+I_{\infty}
$$

then we obtain a real number that equals $\int_{\partial \Omega} u$ classically. Suppose that, with this definition,

$$
\int_{\partial \Omega} u \neq \int_{\Omega} \nabla \cdot u
$$


Then, since the divergence theorem holds if either $K_{\infty}-\{0\}$ is inhabited or else $K_{\infty}=\{0\}$, we must have

$$
\neg \forall_{n}\left(a_{n}=0\right) \wedge \neg \neg \forall_{n}\left(a_{n}=0\right),
$$

which is absurd. Hence (12) is impossible and therefore, since inequality on $\mathbf{R}$ is tight,

$$
\int_{\partial \Omega} u=\int_{\Omega} \nabla \cdot u
$$

In this sense, the divergence theorem, suitably tweaked, holds for bounded, uniformly continuous functions on $\bar{\Omega}$.

In view of (h), we should be aware that, although in the second half of the proof of Theorem 4 the function $u: x \rightsquigarrow \log \|x\|$ is defined throughout $\bar{\Omega}$, we can neither prove it uniformly continuous, nor produce a bound for it, on $\bar{\Omega}$ unless and until we know that there exists $n$ with $a_{n}=1-a_{n-1}$ (or, availing ourselves the use of Markov's principle, unless and until we know that it is impossible that $a_{n}=0$ for all $n$ ).

\section{A limiting result without Markov's principle}

In Theorem 4 we make use of Markov's principle. Since this principle is independent of, but compatible with, BISH, the natural question is of course what we may recover without this principle. The following construction and the propositions that follow it shed some light on the limitations that face us.

Lemma 8 Let $\left(a_{n}\right)_{n \geq 1}$ be a binary sequence. Let $D$ be the open unit disc in $\mathbf{R}^{2}$ and for each positive integer $n$ define

$$
P_{n}=\left\{x \in \mathbf{R}^{2}:\|x\|<1 \wedge 0 \leq \arg x \leq \frac{1}{n}\right\}
$$

where $\arg x$ is the argument of $x$ when $x$ is given in the usual polar coordinates. If $a_{n}=0$, then set $\Omega_{n} \equiv D$. If $a_{n}=1$, then set $\Omega_{n} \equiv D \backslash P_{n}$. Note that for each $n, \Omega_{n}$ is totally bounded. Define

$$
\Omega=\left(\bigcap_{n \geq 1} \Omega_{n}\right)^{\circ}
$$

Then $\Omega$ is totally bounded and integrable. 
Proof We show that $\Omega$ is totally bounded, using an argument similar to that from Lemma 6. Given $\varepsilon>0$, choose a positive integer $N>3 / \varepsilon$. Either there is a (least) $m \leq N$ such that $a_{m}=1$ or $a_{m}=0$ for each $m \leq N$. In the former case, $\Omega=\Omega_{m}$ and an $\varepsilon$-approximation to $\Omega_{m}$ is also an $\varepsilon$-approximation to $\Omega$. In the latter case, note that $\Omega_{N} \subset \Omega$ and choose a finite $\varepsilon / 3$-approximation $\left\{x_{1}, x_{2}, \ldots, x_{n}\right\}$ to $\Omega_{N}$. Consider $x \in D$. Either $|\arg x|>1 / N$ or $|\arg x|<2 / N$. In the first case, $x \in \Omega_{N}$, so there exists $i$ with $\left\|x-x_{i}\right\|<\varepsilon$. In the second case,

$$
\rho\left(x, \Omega_{N}\right)<\sin \left(\frac{2}{N}\right)<\frac{2}{N}<\frac{2 \varepsilon}{3},
$$

so, choosing $y \in \Omega_{N}$ so that $\|x-y\| \leq 2 \varepsilon / 3$ and $i$ such that $\left\|y-x_{i}\right\|<\varepsilon / 3$ we have $\left\|x-x_{i}\right\|<\varepsilon$. Hence $\left\{x_{1}, x_{2}, \ldots, x_{n}\right\}$ is a finite $\varepsilon$-approximation to $D$ with points from $\Omega_{N} \subset \Omega$; therefore, since $\Omega \subset D$, it is an $\varepsilon$-approximation to $\Omega$.

A proof analogous to that in Lemma 6 shows that, like the annular domain constructed there, this domain is integrable.

The construction is illustrated in Fig. 2.
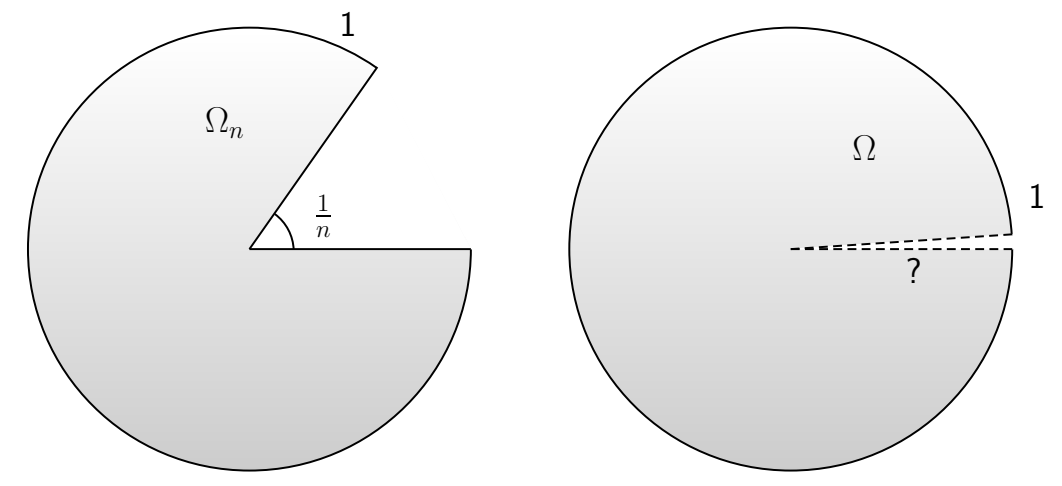

Figure 2: The domains $\Omega_{n}$ (left) when $a_{n}=1$, and the domain $\Omega$ (right), constructed in Lemma 8.

Proposition 9 If for every totally bounded, Lebesgue integrable, open subset $\Omega$ of $\mathbf{R}^{n}$, and every uniformly continuous function $f: \partial \Omega \rightarrow \mathbf{R}$, the Dirichlet problem has a strong solution in $H_{0}^{2}(\bar{\Omega})$, then WLPO holds.

Proof Let $\Omega$ be as in Lemma 8. Suppose that the Dirichlet problem

$$
\Delta u=0 \text { on } \Omega, u(x)=1-\|x\| \text { for all } x \in \partial \Omega
$$


has a strong solution on $\bar{\Omega}$ (it is not difficult to see that the mapping $x \rightsquigarrow 1-\|x\|$ is uniformly continuous on $\partial \Omega$ ). Then we may determine whether $u(0)<1$, in which case $0 \notin \partial \Omega$ and $a_{n}=0$ for all $n$, or $u(0)>0$, in which case it is impossible that $a_{n}=0$ for all $n$; thus WLPO holds.

This leads us to the observation that the integrability of the boundary of an integrable domain is essentially nonconstructive, as embodied by the following result:

Proposition 10 If every open, Lebesgue integrable domain in $\mathbf{R}^{n}$ has an integrable boundary, then WLPO holds.

Proof Let $\Omega$ be as in Lemma 8; so $\Omega$ is integrable. Without loss of generality, let $a_{1}=0$. Suppose that $\partial \Omega$ is integrable. Either $\int_{\partial \Omega} d x<2 \pi+1$, in which case $a_{n}=0$ for all $n$ (and in fact $\Omega=D$ ), or else $\int_{\partial \Omega} d x>2 \pi$ and it is impossible that $a_{n}=0$ for all $n$.

Note that, like the domain from Lemma 6 , the domain $\Omega$ from Lemma 8 has the following properties. The proofs require only minor modifications, though without the use of Markov's principle; see the discussion following comments (c)-(f) on pages $14-16$.

$\left(\mathrm{a}^{\prime}\right)$ The domain $\Omega$ satisfies a pointwise exterior cone condition, but if it satisfies a uniform one, then (9) holds.

$\left(\mathrm{b}^{\prime}\right)$ The domain $\Omega$ is edge coherent.

$\left(\mathrm{c}^{\prime}\right)$ If $\Omega$ is approximated internally by compact sets, then (9) holds.

$\left(\mathrm{d}^{\prime}\right)$ If $\partial \Omega$ is totally bounded, then (9) holds.

$\left(\mathrm{e}^{\prime}\right)$ Although $\Omega$ is located, if $\partial \Omega$ is located then (9) holds.

$\left(\mathrm{f}^{\prime}\right)$ If $(\partial \Omega)_{r}$ is integrable, then (9) holds.

$\left(\mathrm{h}^{\prime}\right)$ The divergence theorem, tweaked as per comment $(\mathrm{h})$ above, holds for uniformly continuous functions on $\bar{\Omega}$.

Thus if, in any of (a) and (c)-(f), the relevant property holds independently of the binary sequence $\left(a_{n}\right)_{n \geq 1}$ used to define $\Omega$, then WLPO is derivable in BISH.

One of the reservations that applied to the annular domain from Lemma 6 concerning the divergence theorem applies here too: with the boundary condition function $f: x \rightsquigarrow$ $1-\|x\|$, we cannot necessarily produce candidate functions for the solution. However, any candidate solution function $u$ is certainly bounded. 
It remains an open question whether the existence of weak solutions suffice for the conclusion of Proposition 9. Further, Proposition 9 leaves open the question whether WLPO is sufficient to construct solutions to the Dirichlet problem. We conjecture that the existence of solutions to Dirichlet-type problems requires strong computability conditions on the boundary such as its locatedness (which condition is classically trivial). Since WLPO is not sufficient to locate sets in $\mathbf{R}^{n}$ in general, it is unlikely that it would be sufficient for existence of solutions to the Dirichlet problem.

Finally, since solutions to the Dirichlet problem are solutions to a special case of the Navier-Stokes equations of fluid flow, we have the following corollary, indicating that any proof of existence of solutions to these equations will have to be non-constructive.

Corollary 11 There is no universal algorithm for computing solutions to the classical Navier-Stokes equations of fluid flow with arbitrary boundary conditions.

Acknowledgement. The authors would like to thank the anonymous referees for constructive comments on drafts of the paper. This work was partially carried out while the second author was a Postdoctoral Fellow supported by the Department of Mathematics \& Statistics, at the University of Canterbury, Christchurch, New Zealand.

\section{References}

[1] P. Aczel and M. Rathjen, Notes on Constructive Set Theory, Report No. 40, Institut Mittag-Leffler, Royal Swedish Academy of Sciences, 2001; available at http: //www . ml.kva.se/preprints/archive/2000-2001/2000-2001-40.pdf.

[2] K.E. Atkinson and Han Weimin, Theoretical Numerical Analysis: A Functional Analysis Framework, Texts in Applied Mathematics 39, Springer Verlag, Heidelberg, 2009.

[3] E.A. Bishop, Foundations of Constructive Analysis, McGraw-Hill, New York, 1967.

[4] E.A. Bishop and D.S. Bridges, Constructive Analysis, Grundlehren der math. Wiss. 279, Springer Verlag, Heidelberg, 1985.

[5] D.S. Bridges and L.S. Vîţă, Techniques of Constructive Analysis, Universitext, Springer New York, 2006.

[6] D.S. Bridges, F. Richman and Wang Yuchuan, Sets, complements and boundaries, Proc. Koninklijke Nederlandse Akad. Wetenschappen (Indag. Math.) 7(4), 425-445, 1996; doi: 10.1016/S0019-3577(97)89131-8.

[7] D.S. Bridges and Wang Yuchuan, Constructing cutoff functions, New Zealand J. Math. 26, 25-30, 1997. 
[8] D.S. Bridges and Wang Yuchuan, Constructive aspects of the Dirichlet problem, $J$. Univ. Comp. Sci. 3(11), 1148-1161, 1997; doi: 10.3217/jucs-003-11-1148.

[9] D.S. Bridges and Wang Yuchuan, Constructive weak solutions of the Dirichlet problem, J. London Math. Soc. (2) 57, 655-667, 1998; doi: 10.1112/S0024610798006243.

[10] Y.K. Chan, Constructive foundations of potential theory, Pacific J. Math. 71(2) 405-418, 1977; doi: 10.2140/pjm.1977.71.405.

[11] M.A.E. Dummett, Elements of Intuitionism (second edition), Oxford Logic Guides 39, Clarendon Press, Oxford, 2000.

[12] L.C. Evans, Partial Differential Equations, Grad. Studies in Math. 19, Amer. Math. Soc. Providence, RI, 1998.

[13] C.L. Fefferman, Existence and smoothness of the Navier-Stokes equation, Clay Mathematics Institute, http://www.claymath.org/millennium/Navier-Stokes_ Equations/navierstokes.pdf.

[14] F. John, Partial Differential Equations, (third edition), Applied Mathematical Sciences 1, Springer-Verlag, Heidelberg-Berlin-New York, 1971.

[15] B.A. Kushner, Lectures on Constructive Mathematical Analysis, Amer. Math. Soc., Providence RI, 1985.

[16] P. Martin-Löf, Intuitionistic Type Theory, Notes by G. Sambin, Bibliopolis, Napoli, Italy, 1984; available at http://www.cs.cmu.edu/afs/cs/Web/People/crary/ 819-f09/Martin-Lof80.pdf.

[17] J. Rauch, Partial Differential Equations, Grad. Texts in Math. 128, Springer Verlag, Heidelberg, 1991.

[18] Wang Yuchuan, Constructive Analysis of Partial Differential Equations, D.Phil. thesis, University of Waikato, Hamilton, New Zealand, 1996; http:// waikato. lconz . ac .nz/vwebv/holdings Info?bibId=319623. Earlier draft available at http://www . math. canterbury.ac.nz/ d.bridges/theses/complete_ Wang_thesis_180213.pdf.

University of Canterbury, Christchurch, New Zealand

D.Bridges@math.canterbury.ac.nz, maarten.jordens@canterbury.ac.nz

Received: 8 December $2011 \quad$ Revised: 17 October 2012 